\title{
Excessive Oxygen Uptake during Exercise and Recovery in Heavy Exercise
}

\author{
T. YANO, T. YUNOKI, R. MATSUURA, T. ARIMITSU, T. KIMURA
}

Exercise Physiology, Graduate School of Education, Hokkaido University, Kita-ku, Sapporo, Japan

Received October 31, 2006

Accepted January 10, 2007

On-line available February 8, 2007

\begin{abstract}
Summary
The aim of this study was to determine whether excessive oxygen uptake $\left(\mathrm{Vo}_{2}\right)$ occurs not only during exercise but also during recovery after heavy exercise. After previous exercise at zero watts for $4 \mathrm{~min}$, the main exercise was performed for $10 \mathrm{~min}$. Then recovery exercise at zero watts was performed for $10 \mathrm{~min}$. The main exercises were moderate and heavy exercises at exercise intensities of $40 \%$ and $70 \%$ of peak $\dot{\mathrm{V}}_{\mathrm{o}_{2}}$, respectively. $\dot{\mathrm{V}}_{\mathrm{o}_{2}}$ kinetics above zero watts was obtained by subtracting $\dot{\mathrm{V}}_{2}$ at zero watts of previous exercise $\left(\Delta \dot{\mathrm{V}}_{2}\right) . \Delta \dot{\mathrm{V}}_{2}$ in moderate exercise was multiplied by the ratio of power output performed in moderate and heavy exercises so as to estimate the $\Delta \dot{\mathrm{Vo}}_{2}$ applicable to heavy exercise. The difference between $\Delta \dot{V}_{o_{2}}$ in heavy exercise and $\Delta \dot{V}_{o_{2}}$ estimated from the value of moderate exercise was obtained. The obtained $\dot{\mathrm{V}}_{2}$ was defined as excessive $\dot{\mathrm{V}}_{2}$. The time constant of excessive $\dot{\mathrm{V}}_{2}$ during exercise $(1.88 \pm 0.70 \mathrm{~min})$ was significantly shorter than that during recovery $(9.61 \pm 6.92 \mathrm{~min})$. Thus, there was excessive $\dot{\mathrm{Vo}}_{2}$ during recovery from heavy exercise, suggesting that $\mathrm{O}_{2}$ /ATP ratio becomes high after a time delay in heavy exercise and the high ratio continues until recovery.
\end{abstract}

\section{Key words}

Excessive oxygen uptake $\bullet$ Time constant $\bullet$ Heavy exercise $\bullet$ Moderate exercise

\section{Introduction}

Oxygen uptake $\left(\dot{\mathrm{V}}_{2}\right)$ has been analyzed by the application of a mathematical equation for $\dot{\mathrm{Vo}}_{2}$ kinetics at the onset and offset of constant-load exercise. This analysis has shown that $\dot{\mathrm{Vo}}_{2}$ exponentially increases at the onset of moderate exercise with constant power output (on-fast component), reaches a steady state, and rapidly decreases at the offset of moderate exercise (offfast component) (Paterson and Whipp 1991, Ozyener et al. 2001, Scheuermann et al. 2001). In heavy exercise, $\dot{\mathrm{Vo}}_{2}$ is additionally increased (on-slow component) after the on-fast component (Barstow and Mole 1991, Paterson and Whipp 1991, Barstow et al. 1996, Ozyener et al. 2001, Scheuermann et al. 2001), but $\dot{\mathrm{Vo}}_{2}$ at the offset of heavy exercise shows only an off-fast component (Ozyener et al. 2001, Scheuermann et al. 2001). From these results, it is concluded that $\dot{\mathrm{V}}_{2}$ kinetics in heavy exercise is different at onset and offset and that there was no off-slow component. However, there is an effect of exercise intensity from separation by another method. For example, the time constant obtained by mathematical analysis for $\mathrm{Vo}_{2}$ kinetics is around $0.5 \mathrm{~min}$ when the data are limited to $3 \mathrm{~min}$ only, but the time constant becomes longer when data for 6 min are used (Paterson and Whipp 1991). Furthermore, it has been reported that $\dot{\mathrm{Vo}}_{2}$ at 
$3 \mathrm{~min}$ during recovery increased in relation to blood lactate level even if the off component extracted by a mathematical equation is one (Yano et al. 2004). Thus, it is likely that there is an excessive factor in $\dot{\mathrm{Vo}}_{2}$ kinetics during recovery from heavy exercise.

Results obtained by using mean power frequency (MPF) of an electromyogram (EMG) to study the kinetics of EMG discharge have suggested that progressive recruitment of fast-twich fibers occurs during the on-slow component (Borrani et al. 2001). In this case, additional motor units could make an oxygen deficit during exercise and could repay oxygen debt during recovery. Indeed, $\dot{\mathrm{Vo}}_{2}$ at the offset of heavy exercise is regarded as oxygen debt in relation to oxygen deficit at the onset of heavy exercise (Paterson and Whipp 1991, Bearden and Moffatt 2000). However, it has been reported that in two repeated bouts, amplitude of the onslow component is reduced in the second bout but that MPF does not change during the two bouts (Scheuermann et al. 2001). This suggests that the on-slow component is due to factors other than recruitment of motor units.

We hypothesized that the efficiency of aerobic energy supply becomes low and that the low efficiency continues during recovery. To test this hypothesis, we examined whether excessive $\mathrm{V}_{\mathrm{O}_{2}}$ exists not only during exercise but also during recovery.

\section{Methods}

Six healthy males with a mean age of $26 \pm 1.9$ years, a mean body weight of $62.9 \pm 4.3 \mathrm{~kg}$, a mean height of $170 \pm 5.3 \mathrm{~cm}$ and a mean peak $\dot{\mathrm{Vo}}_{2}$ of $2.69 \pm 0.14 \mathrm{l} / \mathrm{min}$ participated in this study. After the objective and procedure of the experiment and the risks associated with the experiment were explained, written consent to participate in the study was obtained from each subject. This study was approved by the local ethics committee.

A cycle ergometer in which the power output can be adjusted by a computer (232C, Combi, Japan) was used. On the first day, each subject performed incremental-load exercise after a 5-min rest period to determine his peak $\dot{\mathrm{V}}_{2}$. After cycling at a work rate of zero watts for $4 \mathrm{~min}$, the power output was increased in ramp mode by 15 watts per min until the subject could no longer maintain a rotation rate of $50 \mathrm{rpm}$. On different days, moderate and heavy constant-load exercises were performed for $10 \mathrm{~min}$ after exercise at zero watts for $5 \mathrm{~min}$ and then recovery exercise at zero watts for $10 \mathrm{~min}$. The moderate and heavy exercises were performed at exercise intensities of $40 \%$ and $70 \%$ of peak $\dot{\mathrm{V}}_{2}$, respectively.

$\mathrm{Vo}_{2}$ was measured breath-by-breath using a respiratory gas analyzer (AE-280S Minato Medical Science, Japan). The flow volumes of inspiration and expiration were determined using a hot-wire respiratory meter. The flow signals were integrated electrically for each breath and converted to ventilation per minute. The respiratory meter was calibrated using a 2-liter syringe. The results of measurement using this instrument were linear with ventilation in the range of $0-600 \mathrm{l} / \mathrm{min} . \mathrm{O}_{2}$ and $\mathrm{CO}_{2}$ concentrations were analyzed using a zirconium sensor and infrared absorption analyzer, respectively. The data of $\dot{\mathrm{V}}_{2}$ were followed every 15 seconds.

$\dot{\mathrm{VO}}_{2}$ kinetics above zero watts were obtained by subtracting $\dot{\mathrm{Vo}}_{2}$ at zero watts $\left(\Delta \dot{\mathrm{Vo}}_{2}\right) . \Delta \dot{\mathrm{Vo}}_{2}$ in moderate exercise was multiplied by the ratio of power outputs in heavy exercise (P70) and moderate exercise (P40) to estimate the $\Delta \dot{\mathrm{Vo}}_{2}$ applicable to heavy exercise (Fig. 1). Thus, the estimated $\Delta \dot{\mathrm{Vo}}_{2}$ was obtained by $\Delta \dot{\mathrm{V}}_{2} *(\mathrm{P} 70 / \mathrm{P} 40)$. The difference between $\Delta \dot{\mathrm{V}}_{2}$ in heavy exercise and $\Delta \dot{\mathrm{Vo}}_{2}$ estimated from moderate exercise (see Fig. 2) was obtained. This value was defined as excessive $\mathrm{Vo}_{2}$ (Fig. 3).

$\dot{\mathrm{V}}_{2}$ kinetics at $40 \%$ of peak $\dot{\mathrm{V}}_{2}$ and excessive $\mathrm{Vo}_{2}$ kinetics were approximated by the following equation:

Excessive $\dot{\mathrm{V}}_{2}=\mathrm{A} *(1-\exp (-(\mathrm{t}-\mathrm{TD}) / \tau))$

where $\mathrm{A}$ is the amplitude of the system, $\tau$ is the time constant of the system, $t$ is time and TD is time delay.

The value during recovery was approximated by the following equation:

$\Delta \dot{\mathrm{Vo}}_{2}=\mathrm{A} * \exp (-(\mathrm{t}-\mathrm{TD}) / \tau)$

The value of A obtained by Eq. (1) was used as the value of amplitude in Eq. (2).

Student's t-test (paired samples) was used to test for significance in differences between the variables. The level of significance was set at $\mathrm{P}<0.05$. The results are expressed as means \pm S.D.

\section{Results}

Figure 1 shows the kinetics of $\Delta \dot{\mathrm{Vo}}_{2}$ in moderate exercise and $\Delta \dot{\mathrm{Vo}}_{2}$ estimated from moderate exercise by multiplying by the ratio of power outputs in moderate and heavy exercise. In moderate exercise, $\Delta \dot{\mathrm{Vo}}_{2}$ rapidly increased and showed a steady-state during exercise. The 


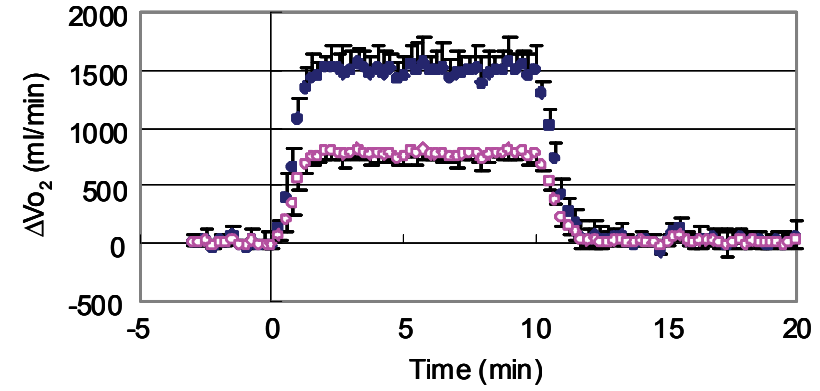

Fig. 1. Oxygen uptake $\left(\dot{\mathrm{V}}_{2}\right)$ above $\dot{\mathrm{V}}_{2}$ at zero watts $\left(\Delta \dot{\mathrm{V}}_{2}\right)$. Moderate exercise was performed from 0 to $10 \mathrm{~min}$. Each subject exercised at zero watts before and after moderate exercise. The open circles show $\Delta \dot{V}_{2}$ in moderate exercise. The closed circles show $\Delta \dot{\mathrm{V}}_{2}$ estimated from $\Delta \dot{\mathrm{V}}_{2}$ in moderate exercise so as to become applicable to $\Delta \dot{V}_{2}$ in heavy exercise.

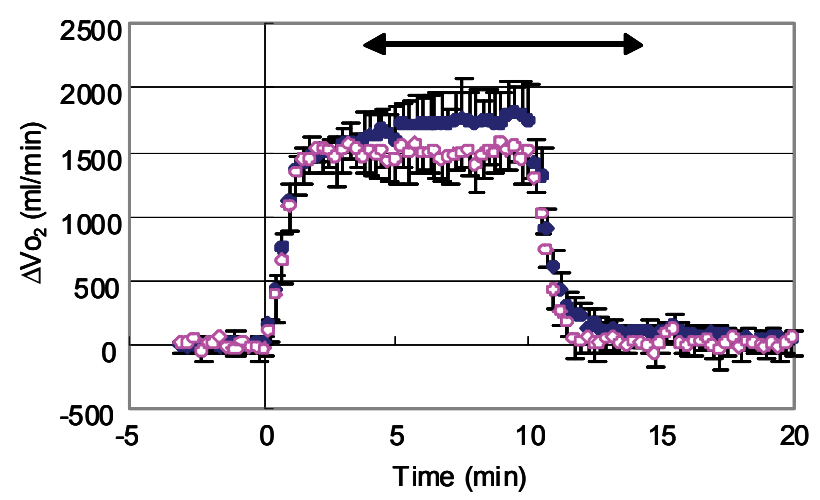

Fig. 2. Oxygen uptake $\left(\dot{\mathrm{V}}_{2}\right)$ above $\dot{\mathrm{V}}_{2}$ at zero watts $\left(\Delta \dot{\mathrm{VO}}_{2}\right)$. Moderate and heavy exercises were performed from 0 to $10 \mathrm{~min}$. Each subject exercised at zero watts before and after moderate and heavy exercises. The closed circles show $\Delta \dot{\mathrm{V}}_{2}$ in heavy exercise, and the open circles show the values estimated from $\Delta \dot{\mathrm{V}}_{2}$ in moderate exercise so as to become applicable to $\Delta \dot{\mathrm{V}}_{2}$ in heavy exercise. The arrow shows a significant difference between $\Delta \dot{\mathrm{V}}_{2}$ estimated from moderate exercise and $\Delta \dot{\mathrm{V}}_{2}$ in heavy exercise.

amplitude was $776 \pm 50.4 \mathrm{ml} / \mathrm{min}$ and the time constant was $0.45 \pm 0.19 \mathrm{~min}$ at the onset of moderate exercise. $\Delta \dot{\mathrm{V}}_{2}$ during recovery rapidly decreased to zero. The time constant at the offset of moderate exercise was $0.58 \pm 0.15$ min and was not significantly different from that at the onset of moderate exercise.

Figure 2 shows $\Delta \mathrm{Vo}_{2}$ kinetics in heavy exercise and $\Delta \dot{\mathrm{V}}_{2}$ estimated from moderate exercise. Since $\Delta \dot{\mathrm{V}}_{2}$ was a value above zero watts, $\Delta \dot{\mathrm{V}}_{2}$ in the previous exercise before moderate and heavy exercises was zero. There were significant differences between $\Delta \dot{\mathrm{Vo}}_{2}$ in heavy exercise and $\Delta \dot{\mathrm{Vo}}_{2}$ estimated from moderate exercise from $4 \mathrm{~min}$ during exercise to $4 \mathrm{~min}$ during recovery.

Figure 3 shows excessive $\dot{\mathrm{V}}_{2}$ in heavy exercise. At the onset of exercise, excess $\dot{\mathrm{V}}_{2}$ showed positive and then negative values. It appeared from these results that

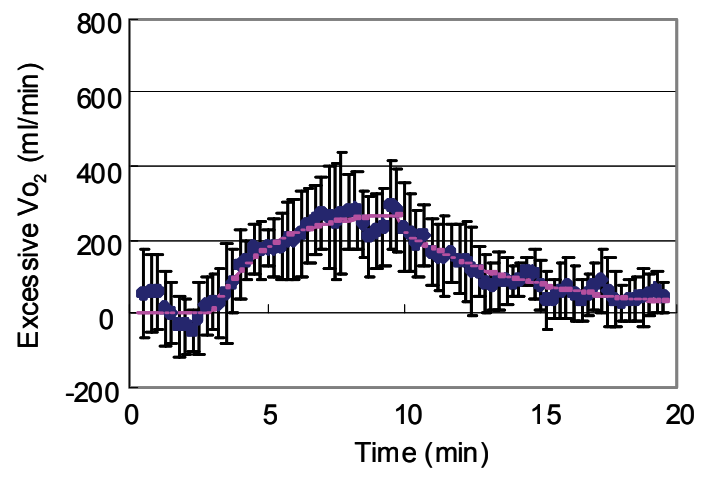

Fig. 3. Excessive oxygen uptake in heavy exercise from 0 to $10 \mathrm{~min}$ and during recovery from 10 to $20 \mathrm{~min}$.

$\Delta \mathrm{Vo}_{2}$ in heavy exercise responded more slowly than that in moderate exercise. However, as shown in Figure 2, the difference between $\Delta \dot{\mathrm{V}}_{2}$ in moderate and heavy exercises is negligible. Excessive $\dot{\mathrm{Vo}}_{2}$ was estimated by equations (1) and (2). The time delay was $2.56 \pm 0.90 \mathrm{~min}$, the time constant was $1.88 \pm 0.70 \mathrm{~min}$ and amplitude was $296 \pm 96 \mathrm{ml} / \mathrm{min}$ during exercise. During recovery, the time constant was $9.61 \pm 6.92 \mathrm{~min}$. There was a significant difference between the time constant during exercise and that during recovery.

\section{Discussion}

The aim of this study was to determine whether excessive $\dot{\mathrm{Vo}}_{2}$ kinetics exists not only during heavy exercise but also during recovery. $\mathrm{Vo}_{2}$ kinetics above zero watts was obtained by subtracting $\mathrm{Vo}_{2}$ at zero watts of previous exercise $\left(\Delta \dot{\mathrm{Vo}}_{2}\right) \cdot \Delta \dot{\mathrm{V}}_{2}$ in moderate exercise was multiplied by the ratio of power outputs in moderate and heavy exercises. The difference between $\Delta \dot{\mathrm{V}}_{2}$ in heavy exercise and $\Delta \dot{\mathrm{Vo}}_{2}$ estimated from moderate exercise was defined as excessive $\dot{\mathrm{V}}_{2}$. There were excessive $\dot{\mathrm{V}}_{2}$ kinetics not only during the exercise but also during recovery after heavy exercise.

Before obtaining excessive $\dot{\mathrm{V}}_{2}, \dot{\mathrm{V}}_{2}$ was subtracted from $\dot{\mathrm{Vo}}_{2}$ at zero watts. Thus, $\dot{\mathrm{Vo}}_{2}$ at zero watts was used for the baseline. It is not certain whether the baseline is $\dot{\mathrm{V}}_{2}$ at zero watts or at rest. However, during cycle exercise at zero watts, the legs are moving. Energy is required for this motion. This can be called internal work (Margaria 1976). This internal work accompanies external work by the cycle ergometer. Therefore, we chose $\dot{\mathrm{Vo}}_{2}$ at zero watts as the baseline to reduce the effect of internal work.

In the present study, $\dot{\mathrm{V}}_{2}$ was separated into two factors by the difference between $\Delta \dot{\mathrm{V}}_{2}$ in heavy exercise and $\Delta \dot{\mathrm{Vo}}_{2}$ estimated from $\Delta \dot{\mathrm{Vo}}_{2}$ in moderate exercise so 
as to make it applicable to $\Delta \dot{\mathrm{V}}_{2}$ in heavy exercise. Therefore, the estimated $\Delta \dot{\mathrm{V}}_{2}$ is attributed to the characteristic of $\dot{\mathrm{Vo}}_{2}$ kinetics in moderate exercise, and the remaining value is attributed to the characteristic of $\dot{\mathrm{V}}_{2}$ kinetics in heavy exercise.

The relationship between oxygen deficit and oxygen debt in heavy exercise has been examined. Paterson and Whipp (1991) reported that the oxygen deficit related to the on-fast component is equivalent to oxygen debt. Bearden and Moffatt (2000) reported that when the oxygen deficit in heavy exercise is the sum of oxygen deficit related to the on-fast component and oxygen deficit related to the on-slow component, its sum is equivalent to the oxygen debt. However, the present results do not support the concept of oxygen debt and deficit in heavy exercise. Since the time constant in excessive $\dot{\mathrm{V}}_{2}$ during exercise was shorter than that during recovery, oxygen debt must be larger than oxygen deficit in relation to excessive $\dot{\mathrm{Vo}}_{2}$ kinetics.

It has been suggested that there are two factors associated with the on-slow component within active muscle (Zoladz and Korzeniewski 2001). One is related to the decrease in efficiency of the ATP-producing system, especially mitochondrial oxidative phosphorylation (increase in $\mathrm{O}_{2} / \mathrm{ATP}$ ratio), and the other is related to the decrease in efficiency of the contractile machinery using ATP (increase in ATP/work rate ratio). Scheuermann et al. (2001) reported that the on-slow component is reduced in the second bout of two repeated bouts of heavy exercise, but the mean power frequency of a surface electromyogram is not changed during the two repeated bouts. They suggested that these results are associated with an increase in ATP requirements of the already recruited motor units rather than changes in the recruitment pattern of slow versus fast-twitch motor units. Accordingly, an increase in ATP/work rate ratio is likely to be a cause of the on-slow component. However, they did not examine the off-slow component because in their mathematical analysis only one off-component was extracted during recovery from heavy exercise. However, the present results showed that there was excessive $\dot{\mathrm{V}}_{2}$ not only during exercise but also during recovery. This result obtained during recovery cannot be explained by an increase in ATP/work rate ratio because excessive $\dot{\mathrm{Vo}}_{2}$ that is induced by an increase in ATP/work rate ratio should be minimum during recovery since ATP is less required for work at zero watts. Therefore, excessive $\dot{\mathrm{V}}_{2}$ is thought to be associated with increase in $\mathrm{O}_{2} /$ ATP ratio.

\section{Conclusion}

From the results obtained by using the present separation method, it is concluded that excessive $\dot{\mathrm{V}}_{2}$ remains during recovery from heavy exercise. This suggests that the $\mathrm{O}_{2}$ /ATP ratio becomes high after a time delay in heavy exercise and that the high ratio continues until recovery.

\section{References}

BARSTOW TJ, MOLE PA: Linear and nonlinear characteristics of oxygen uptake kinetics during heavy exercise. J Appl Physiol 71: 2099-2106, 1991.

BARSTOW TJ, JONES AM, NGUYEN PH, CASABURI R: Influence of muscle fiber type and pedal frequency on oxygen uptake kinetics of heavy exercise. $J$ Appl Physiol 81: 1642-1650, 1996.

BEARDEN SE, MOFFATT RJ: $\dot{\mathrm{V}}_{2}$ kinetics and the $\mathrm{O}_{2}$ deficit in heavy exercise. $J$ Appl Physiol 88: 1407-1412, 2000.

BORRANI F, CANDAU R, MILLET GY, PERREY S, FUCHSLOCHER J, ROUILLON JD: Is the $\mathrm{Vo}_{2}$ slow component dependent on progressive recruitment of fast-twitch fibers in trained runners? $J$ Appl Physiol 90: 2212-2220, 2001.

MARGARIA R: Biomechanics and Energetics of Muscular Exercise. Clarendon Press, Oxford, 1976.

OZYENER F, ROSSITER HB, WARD SA, WHIPP BJ: Influence of exercise intensity on the on- and off-transient kinetics of pulmonary oxygen uptake in humans. J Physiol Lond 533: 891-902, 2001.

PATERSON DH, WHIPP BJ: Asymmetries of oxygen uptake transients at the on- and offset of heavy exercise in humans. J Physiol Lond 443: 575-586, 1991.

SCHEUERMANN BW, HOELTING BD, NOBLE ML, BARSTOW TJ: The slow component of $\mathrm{O}_{2}$ uptake is not accompanied by changes in muscle EMG during repeated bouts of heavy exercise in human. $J$ Physiol Lond 531: 245-256, 2001.

YANO T, OKUYAMA T, REIHAN A, OGATA H: Effect of blood lactate level on oxygen uptake at the offset of middle-intensity exercise. Biol Sport 21: 231-239, 2004. 
ZOLADZ JA, KORZENIEWSKI B: Physiological background of the change point in $\mathrm{Vo}_{2}$ and the slow component of oxygen uptake kinetics. J Physiol Pharmacol 52: 167-184, 2001.

\section{Corresponding author}

T. Yano, Exercise Physiology, Graduate School of Education, Hokkaido University, Kita-ku, Sapporo 060-0811, Japan. Fax: 011-706-4943. E-mail: yano@edu.hokudai.ac.jp 